\title{
The Preparation of Transparent Silicone Rubber with Rapid Vulcanization and Ultraviolet Absorbing Effect
}

\author{
Jia Liya, Man Zhongbiao \\ Elkem Silicones Shanghai Co., Ltd., Shanghai, China \\ Email address: \\ liya.jia@elkem.com (Jia Liya)

\section{To cite this article:} \\ Jia Liya, Man Zhongbiao. The Preparation of Transparent Silicone Rubber with Rapid Vulcanization and Ultraviolet Absorbing Effect. \\ International Journal of Materials Science and Applications. Vol. 7, No. 4, 2018, pp. 142-146. doi: 10.11648/j.ijmsa.20180704.14
}

Received: May 28, 2018; Accepted: July 23, 2018; Published: August 17, 2018

\begin{abstract}
Silicone materials have excellent weathering resistance, high and low temperature resistance, anti-yellowing and a series of excellent properties over other materials. In this study vinyl silicone oil as the base polymer, hydrogen silicone oil as a cross-linking agent, vinyl silicone resin and ultraviolet absorber as additives, platinum complex as a catalyst, were used to make ultraviolet absorption of highly transparent silicone rubber. Different structures of silicone resin with vinyl group have different effects on the curing speed of the products. The silicone resin with $\mathrm{MM}^{\mathrm{Vi}} \mathrm{Q}$ can help achieve rapid curing speed. Due to polarity difference for polysiloxane system and ultraviolet absorber, when necessary a suitable organic solvent may be introduced to increase the compatibility of the ultraviolet absorber with the silicone composition to prepare transparent products. Considering that the addition of organic solvents may have effects such as volatiles and properties, the amount of solvent introduced should be as low as possible. In addition, regarding the amount of the ultraviolet absorber added, too little ultraviolet absorber may not achieve a satisfactory ultraviolet absorption effect, but too much would lead to an increase in the amount of solvent introduced. Different ultraviolet absorber could induce different ultraviolet absorption effect and aging resistance.
\end{abstract}

Keywords: Vinyl Silicone Resin, Light Transmittance, Ultraviolet Absorber, Rapid Vulcanization

\section{Introduction}

With the rapid development of polymer technology and related research, more and more optical components and their components contain or consist of polymer materials. Compared with traditional inorganic materials, polymer materials not only maintain the excellent performance of traditional inorganic materials, but also have the advantages of light weight, low cost, simple process, and good mechanical properties. Optical polymer materials can be used for optical bonding, made into lenses or optical sheets and substrates, and can be used in the following components: optical films, optical filters, CCD optical systems, photographic imaging equipment, optical communications and waveguides, fiber optics, light Sensors, holographic optics, interferometers, optocouplers, optical lenses such as microlenses, lenticular lenses, prism lenses, Fresnel lenses, and the like [1-3]. Currently available polymers for the preparation of optical components and their components are: polymethyl methacrylate, polyurethane, polycarbonate, polyvinyl chloride, polystyrene, polyester, epoxy, cycloolefin polymers, polysiloxane and so on. Among them, polysiloxane has drawn extensive attention due to its excellent resistance to high and low temperature, weather resistance, ultraviolet resistance, and aging resistance, and has been made into a variety of optical sheets, substrates or optical adhesives. Polymer materials such as polymethyl methacrylate and polyvinyl chloride have high light transmittance and are easy to process. However, they are prone to aging and deformation when they are under excessively cold, overheated, ultraviolet or other radiation environments, which may cause the decreased stability of lens performance and may be useless [4, 5].

Silicone materials have excellent weathering resistance, high and low temperature resistance and anti-yellowing and a series of excellent properties, and have obvious advantages over other materials. It has been widely applied to the preparation of optical components. For example, a 
polysiloxane-based optical material is used in place of conventional polymethyl acrylate to avoid problems such as poor aging resistance, where the silicone material is crosslinked by exposing the uncured polysiloxane composition to an electron beam. However, this method requires special equipment and high cost [6]. Therefore, the development of a polysiloxane composition that can be quickly vulcanized at room temperature or slightly elevated temperature, and the development of a transparent silicone that can absorb light waves (such as ultraviolet light waves) with outstanding aging resistance are of great significance for protecting related devices from ultraviolet damage.

Silicone resin has its outstanding advantages in the preparation of transparent materials, which not only enables the product to have a high degree of transparency, but also has good mechanical properties. In the platinum-catalyzed polyaddition reaction system, in order to obtain a highly transparent silicone material having good mechanical properties, it is very important to add a silicone resin, particularly a silicone resin containing a reactive functional group such as a vinyl group. In the present study, vinyl silicone oil as the base polymer, hydrogen silicone oil as a cross-linking agent, vinyl silicone resin and ultraviolet absorber as additives, platinum complex as a catalyst, were used to make ultraviolet absorption of highly transparent silicone rubber. The influence of silicone resin on the vulcanization of silicone rubber, the influence of ultraviolet absorber on the light transmittance, ultraviolet absorption effect and aging resistance of silicone rubber were discussed.

\section{Experimental}

\subsection{Material}

The following raw materials were from Elkem silicones: vinyl silicone resin $\mathrm{A} 1\left(\mathrm{MM}^{\mathrm{Vi}} \mathrm{Q}\right.$, vinyl content of about $0.041 \mathrm{~mol} / 100 \mathrm{~g})$, vinyl silicone resin $\mathrm{A} 2\left(\mathrm{MD}^{\mathrm{Vi}} \mathrm{Q}\right.$, vinyl content of about $0.021 \mathrm{~mol} / 100 \mathrm{~g}$, vinyl-terminated silicone oil A3: (viscosity About $3500 \mathrm{mPa} \cdot \mathrm{s}$ ), vinyl-terminated silicone oil A4 (viscosity about $60000 \mathrm{mPa} \cdot \mathrm{s}$ ), hydrogen-containing silicone oil B1 (hydrosilyl content $0.91 \mathrm{~mol} / 100 \mathrm{~g}$ ), hydrogencontaining silicone oil B2 (hydrosilyl content: $0.19 \mathrm{~mol} / 100 \mathrm{~g}$ ) and H50614. Karstedt catalyst (platinum (0)divinyltetramethyldisiloxane complex) was obtained from Shanghai Heraeus Industrial Technology Materials Co., Ltd. Company. TINUVIN 384-2, Ciba TINUVIN 400 and TINUVIN 292HP were obtained from BASF Corporation. PARSOL GUARD was gotten from DSM; Solvent 1 (PAESTER 9145SD) was bought from Patech Fine Chemicals Co., Ltd). Solvents 2 (Isododecane) was bought from marketing.

\subsection{Equipment}

High Speed Mixer: DAC 600.1 FVZ, HAUSCHILD; Ultraviolet Spectrophotometer: UV -2600, Shimadzu Corporation, Japan; Servo control system universal testing machine: AI-7000S, High Speed Rail Testing Instrument Company; Hardness Tester: H04.3130.000, Zwick Corporation; Viscometer: PROGRAMMABLE DV-II+, Brookfield.

\subsection{Preparation of Silicone Rubber}

According to the recipe, the vinyl silicone resin, the vinyl terminated silicone oil, inhibitor and the platinum complex catalyst were stirred at $2000 \mathrm{r} / \mathrm{min}$ for 3 times with a high-speed stirrer and mixed uniformly for 20 second each time.

After mixing well, hydrogen-containing silicone oil and ultraviolet absorber diluted with solvent when necessary were added and stirred 3 times at $2000 \mathrm{r} / \mathrm{min}$ in a high-speed stirrer and mixed well for 20 second each time. The above mixture was vulcanized at $45^{\circ} \mathrm{C}$ to give a silicone rubber. After curing, the samples were stored at $150^{\circ} \mathrm{C}$ for 24 hours and their aging transmittance was measured.

\subsection{Performance Testing and Characterization}

Appearance: the composition was vulcanized at low temperature for 1 hour at $45^{\circ} \mathrm{C}$, and the appearance of the vulcanized sample (with diameter of about $30 \mathrm{~mm}$ and thickness of $6 \mathrm{~mm}$ ) thereof was observed visually. Transmittance: a vulcanized sample (about $50 \mathrm{~mm}$ length, $20-30 \mathrm{~mm}$ of width and thickness of $1 \mathrm{~mm}$ ) was prepared, and the transmittance at $450 \mathrm{~nm}$ was measured with an ultraviolet spectrophotometer; Absorption performance: the same sample preparation and test methods as the light transmittance were used to obtain the relevant light transmittance curves. Viscosity: tested in accordance with ASTM D445. Hardness: tested in accordance with ASTM D2240; Tensile Strength, Elongation at Break: tested in accordance with ASTM D412; Tear Strength: tested in accordance with ASTM D624A; Vulcanization conditions: $45^{\circ} \mathrm{C}$ for 1 hour.

\section{Results and Discussion}

\subsection{Effect of Resin Type on Vulcanization Performance of Silicone Rubber}

The silicone resin may be made of a siloxane unit $M$ selected from the formula $\mathrm{R}^{3} \mathrm{SiO}_{1 / 2}$, a siloxane unit $\mathrm{D}$ of the formula $\mathrm{R}^{2} \mathrm{SiO}_{2 / 2}$, a siloxane unit $\mathrm{T}$ of the formula $\mathrm{RSiO}_{3 / 2}$ and a siloxane unit $\mathrm{Q}$ of the formula $\mathrm{SiO}_{4 / 2}$, etc. Here $\mathrm{R}$ generally represents a monovalent hydrocarbon group having 1 to 20 carbon atoms and at least one of these siloxane units is a siloxane unit $\mathrm{T}$ or $\mathrm{Q}$. $\mathrm{R}$ is methyl in this experiment. In some silicone structures, at least one of the siloxane units $\mathrm{M}, \mathrm{D}$, and $\mathrm{T}$ may comprise an alkenyl group. When this experiment refers to a unit T, Q or D, the unit with the superscript "Vi" represents a unit containing a vinyl group. A polyorganosiloxane resin such as $\mathrm{MM}^{\mathrm{Vi}} \mathrm{Q}$, which is mainly composed of a monovalent siloxane unit $\mathrm{M}$ of the formula $\mathrm{R}^{3} \mathrm{SiO}_{1 / 2}$, and a monovalent siloxane unit $\mathrm{M}^{\mathrm{Vi}}$ of the formula $\mathrm{R}^{\prime} \mathrm{R}^{2} \mathrm{SiO}_{1 / 2}$, and a tetravalent siloxane unit $\mathrm{Q}$ of the formula $\mathrm{SiO}_{4 / 2}$. Here $\mathrm{R}^{\prime}$ represents vinyl 
group. An organopolysiloxane resin of the formula $M D^{\mathrm{Vi}} \mathrm{Q}$, which is substantially composed of a divalent siloxane unit $\mathrm{D}^{\mathrm{Vi}}$ of the formula RR'SiO ${ }_{2 / 2}$, a monovalent siloxane unit $\mathrm{M}$ of the formula $\mathrm{R}^{3} \mathrm{SiO}_{1 / 2}$, and a tetravalent siloxane unit of the formula $\mathrm{SiO}_{4 / 2}$.

Silicone rubber is usually reinforced by loading silica, but silica often causes a reduction in the transparency of the product. Adding vinyl silicone resin can not only significantly increase the mechanical properties of silicone rubber, but also does not affect the transparency of the product. Increasing the quantity of the silicone resin, the mechanical properties of the product will increase. However too much silicone resin is added, the viscosity of the product will be higher and could affect the operating performance of the product, sometimes the product will also become hard and brittle. Therefore, the amount of silicone resin to be added is usually based on the requirements of the operation and mechanical properties [7-15].

Table 1. Composition of silicone rubbe.

\begin{tabular}{llll}
\hline \multirow{2}{*}{ Raw materials/weight parts } & Experiment number & F3 \\
\cline { 2 - 4 } & F1 & F2 & 22 \\
\hline A1 & 22 & 22 & 67 \\
A2 & & 67 & 11 \\
A3 & 67 & 11 & 30 \\
A4 & 11 & 0.03 & 4.9 \\
H50614 & 0.03 & 30 & 4 \\
Karstedt catalyst $\left(\times 10^{-6}\right)$ & 30 & 4.9 & 2.7 \\
B1 & 6.5 & 4 & 2.6 \\
B2 & 5.4 & & \\
Mole ratio of SiH/Vinyl & 2.6 & & \\
\hline
\end{tabular}

Table 2. Properties for silicone rubber.

\begin{tabular}{llll}
\hline \multirow{2}{*}{ Testing items } & Experiment number & & \\
\cline { 2 - 4 } & F1 & F2 & F3 \\
\hline appearance & transparent & transparent & transparent \\
viscosity $/ \mathrm{mPa} \cdot \mathrm{S}$ & 4960 & 3700 & 3700 \\
Refraction index & 1.41 & 1.41 & 1.41 \\
Shore A hardness & 46 & uncured $^{1)}$ & uncured \\
Tensile Strength $/ \mathrm{MPa}$ & 7.1 & - & - \\
Elongation at Break $/ \%$ & 147 & - & - \\
Tear Strength $/ \mathrm{N} \cdot \mathrm{mm}^{-1}$ & 3.6 & - & - \\
\hline
\end{tabular}

Note: 1) flow state after $45^{\circ} \mathrm{C}$ for 1 hour, gel state after $45^{\circ} \mathrm{C}$ for 3 hours

Different composition of silicone rubber containing different silicone resin could be found in Table 1 and related testing results were showed in Table 2. The result indicated vinyl silicone resins with different structures like $\mathrm{MM}^{\mathrm{Vi}} \mathrm{Q}$ and $\mathrm{MD}^{\mathrm{Vi}} \mathrm{Q}$ can make transparent product. Despite the same mass of vinyl silicone oil and vinyl silicone resin and the same mole ratio of $\mathrm{SiH} /$ vinyl, the composition $\mathrm{F} 1$ had a faster cure rate at $45^{\circ} \mathrm{C}$ and cures more completely. For the composition F2 it could not cure at the same condition. Even if the curing inhibitor H 50614 was REMOVED from the F3 formula, it still showed a slower cure rate. The probable reason was that the position of vinyl groups in the silicone resin had a significant effect on the vulcanization rate of the final product, i.e. $M M^{\mathrm{Vi}} \mathrm{Q}$ indicated faster reaction speed than $M D^{\mathrm{Vi}} \mathrm{Q}$.

\subsection{Effect of Ultraviolet Absorber Types on Transmittance and Ultraviolet Absorption of Silicone Rubber}

Table 3 showed the light transmittance of composition containing different ultraviolet absorbers and their corresponding vulcanizates. When ultraviolet absorbers were added, the groups contained in the ultraviolet absorbers generally has strong polarity, and the polysiloxane system was with less polarity, so the compatibility between ultraviolet absorber and polysiloxane system was often poor. The simple ultraviolet absorber loading into polysiloxane system could easily cause decreased transparency. Therefore, when necessary a suitable organic solvent may be introduced to increase the compatibility of the ultraviolet absorber with the silicone composition. Considering that the addition of organic solvents may have effects such as volatiles and properties, the amount of solvent introduced should be as low as possible. In addition, regarding the amount of the ultraviolet absorber added, too little ultraviolet absorber may not achieve a satisfactory ultraviolet absorption effect, but too much would lead to an increase in the amount of solvent introduced. Under the premise of ensuring the ultraviolet absorption effect, the amount of the ultraviolet absorber was generally not more than $3 \%$, preferably less than $1 \%$. Mostly the vulcanized silicone rubbers with different ultraviolet absorbers exhibited high light transmittance in the presence of a small amount of solvents. While F5 formulas had poor compatibility for Ciba TINUVIN 400 and polysiloxane systems, more solvents were added to improve compatibility; excessive solvent addition caused the solvent to precipitate from the cured sample, resulting in low light transmission. 
Table 3. Silicone rubber formulas containing different ultraviolet absorbers.

\begin{tabular}{|c|c|c|c|c|c|}
\hline \multirow{2}{*}{ Composition/weight parts } & \multicolumn{5}{|c|}{ Experiment number } \\
\hline & F1 & F4 & F5 & F6 & F7 \\
\hline F1 & 100 & 100 & 100 & 100 & 100 \\
\hline TINUVIN 384-2 & & 0.47 & & & \\
\hline Mixture of Ciba TINUVIN 400 and solvent 1 (Ciba TINUVIN 400:solvent $1=1: 334$ ) & & & 18.1 & & \\
\hline Mixture of PARSOL GUARD and solvent 2 (PARSOL GUARD: solvent $2=1: 1$ ) & & & & 1.3 & \\
\hline TINUVIN 292HP & & & & & 0.47 \\
\hline Light transmitrance $/ \%$ & 92 & 93 & 21 & 90 & 91 \\
\hline
\end{tabular}

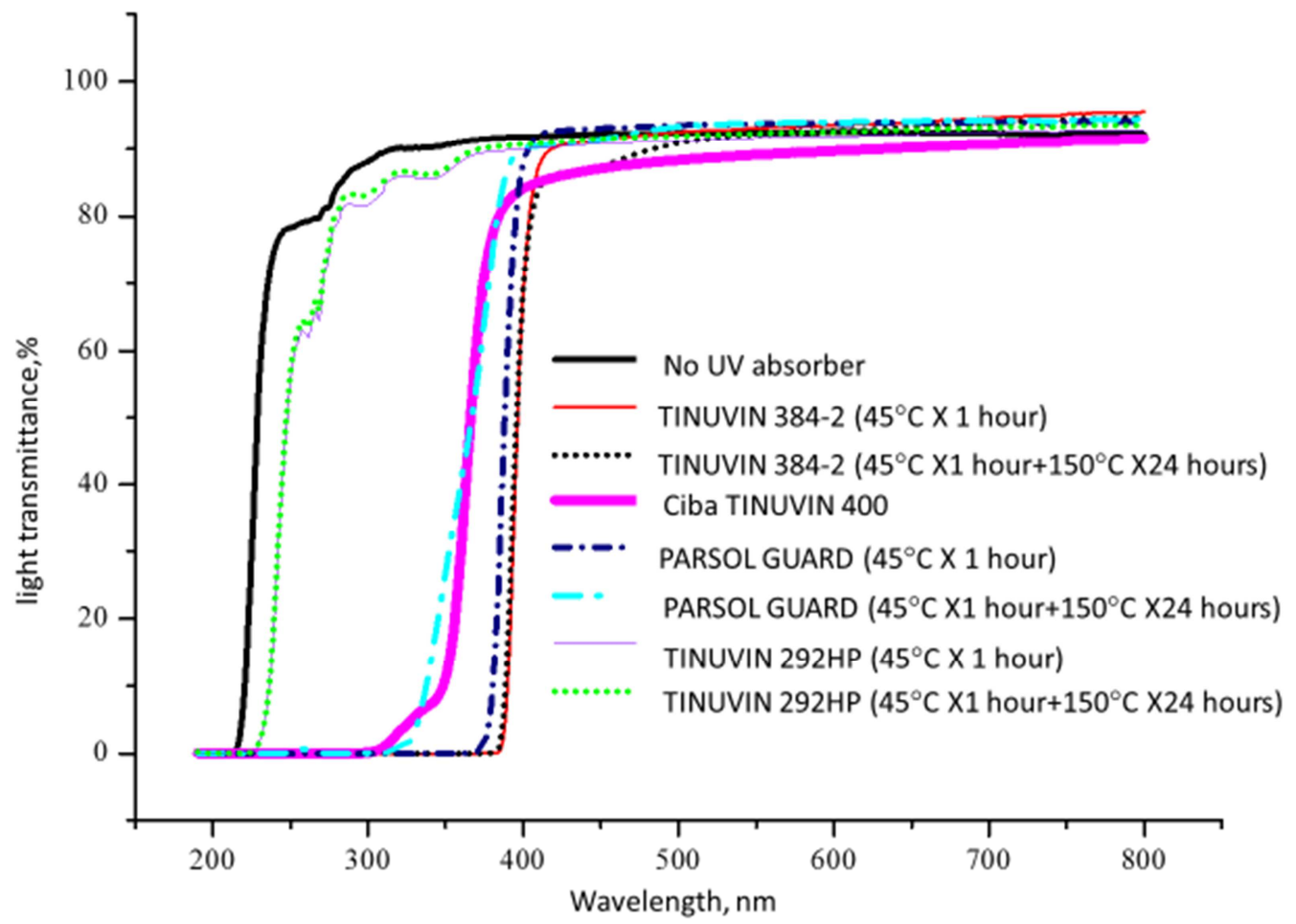

Figure 1. Effect of ultraviolet absorber species on light transmittance of silicone rubber.

Effect of ultraviolet absorber species on light transmittance of silicone rubber could be seen from Figure 1. The vulcanized silicone rubber containing ultraviolet absorber TINUVIN 384-2 had a good light transmittance and ultraviolet absorption effect. Even under the accelerated aging test conditions such as $150^{\circ} \mathrm{C}$ for 24 hours, the vulcanized silicone rubber containing TINUVIN 384-2 still maintained good light transmittance and ultraviolet absorption effect. At the same time, when the ultraviolet absorber TINUVIN 384-2 was used, no additional organic solvent was needed. Silicone rubbers containing TINUVIN 292HP and Ciba TINUVIN 400 ultraviolet absorbers did not completely absorb ultraviolet light. Silicone rubber with TINUVIN 292HP still had no change in light transmittance and ultraviolet absorption after aging. Although the vulcanized silicone rubber containing PARSOL GUARD ultraviolet absorber initially showed better ultraviolet absorption effect, the ultraviolet absorption effect deteriorated after aging at $150^{\circ} \mathrm{C}$ for 24 hours. Therefore, the vulcanized silicone rubber containing the ultraviolet absorber TINUVIN 384-2 was significantly superior to other ultraviolet absorbers in transparency and ultraviolet absorption effects.

\section{Conclusion}

Silicone resin containing a vinyl group was loaded into polyaddition reaction system to prepare transparent silicone rubber. Vinyl silicone resin with $\mathrm{MM}^{\mathrm{Vi}} \mathrm{Q}$ structure helped to prepare fast curing transparent silicone rubber with better mechanical properties. Different ultraviolet absorbers had different effects on ultraviolet absorption. When necessary a suitable organic solvent may be loaded to increase the compatibility of the ultraviolet absorber with the silicone composition to prepare transparent products. The curing composition containing TINUVIN 384-2 ultraviolet absorber could maintain a good light transmittance and ultraviolet absorption effect, also had better aging resistance.

\section{References}

[1] N. Ribeiro, J.-A. Yang, H.-B. Wang, Y.-X. Lin, M. Murakami, T. Unezaki, H. Makio, "Polymerizable Composition for Optical Material and Use Thereof," WO2018070383A1 [P], April 192018. 
[2] J. Park, J.-H. Bae, J. Jung, D. Kang, "Flexible Liquid Crystal Lens," US2018107056A1 [P], April 192018.

[3] J.-H. Lee, S.-J. Park, S.-H. Hwang, B.-J. Mun, H.-S. Song, "Resin Composition for Optical Material and Optical Film Comprising the Same," KR20180018334A [P], February 21 2018.

[4] H. Kametani, S. Ogi, K. Imazumi, N. Kiyoyanagi, J. Ichikawa, T. Mizu, guchi, R. Shitara, "(Meth) acrylate Compound, Resin Composition Containing the Same, Cured Product of the Resin Composition, and Energy Ray-Curable Resin Composition for Optical Lens Sheet and Cured Product thereof,"CN101675089A [P], March 172010.

[5] J. Wang, X.-H. Zhang, X.-M. Zhang, X.-L. Kong, C.-C. Li, E.-L. Xiao, Q. Zhang; L.-P. Sun, PVC "Composition Used for Fresnel Lens and Preparation Method," CN102863709A [P], January 092013.

[6] T. G. Pett, T. J. Hebrink, N.-Y. Jing, J. A. Riddle, D. S. Thompson, A. K. Hartzell, J.-K. Liu, "Silicone-Based Material," CN102918093A [P], February 062013.

[7] S.-M. Xing, Y.-L. Wang, "Organic Silicone Synthesis Technology and Product Application,” p.717, 2000.

[8] A. Pouchelon, F. Lafaysse, "Method for Obtaining a Fibrous Material/Silicone Composite and Said Fibrous Material/Silicone Composite," CN101680162 [P], March 24 2010 .
[9] M. Bahadur, R. Nelson, M. Strong, "Silicone Composition for Producing Transparent Silicone Materials and Optical Devices," CN102414275 [P], April 112012.

[10] K. Nishijima, T. Iimura, M. Suto, T. Sagawa, H. Furukawa, "Curable Silicone Composition, Cured Product Thereof, And Optical Semiconductor Device," CN 105518076 [P], April 20 2016.

[11] T. Yamazaki, T. Kashiwagi, M. Wakao, "Addition-Curable Silicone Resin Composition and Die Attach Material for Optical Semiconductor Device," CN105924974 [P], September 072016.

[12] T. Shiobara, T. Kashiwagi, K. Imazawa, "LEDs-Forming Silicone Resin Composition and Silicone Lens," US7829651 [P], November 092010.

[13] K. Miyoshi, "Silicone Resin Composition for LED Devices," US20040116640 [P], June 172004.

[14] L. L Zhang, Z. C. Li, X. Xu, "Ultraviolet-Curable OrganicSilicon Liquid Optical-Adhesive Composition and Preparation Method of Composition," CN107674640A [P], February 9 2018.

[15] K. Sato, T. Ozai, E. Tabei, "Addition-Curable Silicone Resin Composition, Method for Producing Said Composition, and Optical Semiconductor Device," TW201734135A [P], October 12017. 